\title{
Synthesis of polyanthranilic acid-Au nanocomposites by emulsion polymerization: development of dopamine sensor
}

\author{
BHAVANA GUPTA* ${ }^{*}$, AMBROSE MELVIN $^{1}$ and RAJIV PRAKASH \\ School of Materials Science and Technology, Indian Institute of Technology, Banaras Hindu University, \\ Varanasi 221 005, India \\ ${ }^{1}$ Department of Chemistry, University of Pune, Ganeshkhind, Pune 411 007, India \\ ${ }^{\dagger}$ Present address: Surface and Nanoscience Division, Materials Science Group, \\ Indira Gandhi Centre of Atomic Research, Kalpakkam 603 102, India
}

MS received 16 April 2013; revised 14 October 2013

\begin{abstract}
Polyanthranilic acid (PANA) and polyanthranilic acid-gold (PANA-Au) nanocomposites have been synthesized through emulsion polymerization technique. Use of gold chloride as an oxidant for anthranilic acid not only provides a new route for chemical synthesis of PANA, but also explores a facile method for the formation of nanocomposites. Emulsion polymerization helps in slowing down kinetics of polymerization in comparison to one-phase polymerization and thereby induces formation of monodispersed, both pure and Au nanoparticles, embedded PANA sphere. Reaction progress of nanocomposite formation is studied by UV-Vis spectroscopy for 0-24 h. PANA-Au nanocomposites are characterized by SEM, equipped with EDS, TGA, FT-IR, XRD and electrochemical techniques. XRD of nanocomposites depicts the amorphous nature of polymer and crystalline nature of Au with crystallite size of $\sim 24 \mathbf{~ n m}$. Differential pulse voltammetry has shown the electro-active nature of PANA. The nanocomposites with improved thermal properties show good dispersion in common organic solvents, and it can be explored for application in interference-free dopamine sensors with sensitivity $12.5 \mu \mathrm{A} / \mathrm{mM}$. Acidic group (-COOH) on the polymer makes the sensor free from ascorbic acid interference.
\end{abstract}

Keywords. Polyanthranilic acid; nanocomposite; in situ polymerization; emulsion polymerization; nanoparticles.

\section{Introduction}

Conducting polymer is one of the most applicable materials either in pristine or in modified form, as it exhibit various desirable and switchable electrical, optical and magnetic properties. The proposed applications of conducting polymers are nanoscale electronics (Singh et al 2008; Jin et al 2009; Singh et al 2010), photonics (Nielsen et al 2005), sensors (Banik et al 2008; Mohan and Prakash 2010), and energy storage (Gupta and Prakash 2009). In addition, composite formation is an easiest approach to increase applicability of conducting polymers to a broader range of devices. Among the composite, nanocomposite have recently garnered significant attention because of their unique and synergistic physiochemical property (Mirkin and Letsinger et al 1996; Boal et al 2000; Maheshwari and Saraf et al 2006). Nanocomposites are not only known for their nano-dimension but also for exhibiting synergistic properties due to the combination of its two different components, i.e. matrix and filler, in particular, conducting polymer and metal-based nanocomposites

*Author for correspondence (bgupta1206@gmail.com) formed by decorating a conducting polymer with metal nanoparticles such as Ag, Au, Pt or Pd (Hable and Wrighton 1991; Majumdar et al 2005, 2006; Tseng et al 2005) have been reported for multiple technological application related to catalysis, sensors, electronic devices like transistor, etc. In general, there are two suitable methods of synthesis of conducting polymer metal-based nanocomposite. In the first method, nanoparticle separately synthesized followed by polymerization around the particles or dispersion of the nanoparticle in a polymer matrix (Dewald et al 2005; Mallick et al 2009; Melvin et al 2010), whereas in the second method, both polymerization and reduction of metal ion to nanoparticle takes place simultaneously (Mallicket al 2006; Selvaraj and Alagar 2008; Power et al 2010). Most of the reports related to conducting polymer nanocomposites are dealt with $\mathrm{PANi}-\mathrm{Au}$ and other nonprocessable polymer. In this cases, the evidence of functionalization or co-ordinative interaction between metal and polymer have neither been mentioned nor discussed. Thus, it is required to select a polymer as well as a synthetic approach by which a complexation takes place between the metal and the polymer and that will become more useful for further application. 
Nanoparticle-incorporated conducting polymeric matrices are of recent technological interest in the development of sensor. Amongst various kinds of nanocomposites, special attention has been given to conducting polymers-Au based nanocomposite due its capability in detecting different types of analyte. Dopamine (DA) which is indeed one of the crucial analyte present in extra-cellular fluid of mammalian central nervous system (CNS) with low and varying basal concentration (nano to micromolar range) (Downard et al 1995) shows the difficulty in the voltammetric detection due to ascorbic acid (AA) interference. Conducting polymer-Au matrix is already reported for DA estimation by voltammetric technique. It describes that conducting polymer are responsible for both selective and catalytic oxidation of DA and uric acid. While the enhancement in sensitivity up to nanomolar level occurred due to the presence of $\mathrm{Au}$ particles (Mathiyarasu et al 2008). Some conducting polymers have been used for dopamine sensor without AA interference (Tu et al 2007; Pandey et al 2009). However, there is no report related to conducting polymer with metal nanoparticle, which not only help in the catalytic oxidation of DA, but also repel AA from the close proximity. In this paper, we report an emulsion polymerization route without using any surfactant for the preparation of PANA and PANA-Au nanocomposite for their comparative structural, electrochemical, thermal and morphological properties. Furthermore, the resultant nanocomposite is used to fabricate interference-free dopamine sensor.

\section{Experimental}

\subsection{Materials}

Anthranilic acid was obtained from Rolex India Ltd, India. The monomer was used after recrystallization. Ammonium peroxodisulphate, hydrochloric acid and chloroform were obtained from Merck, India. Gold chloride $\left(\mathrm{HAuCl}_{4}\right)$ from SRL, Sisco India Ltd. All other chemicals used were of analytical grade. Double-distilled deionized water was used in all the experiments.

\subsection{Instruments}

The electrochemical characterizations were carried out using electrochemical workstation (model CHl7041C), $\mathrm{CH}$-Instrument Inc., Texas, USA. Differential pulse voltammetry was recorded in a single-compartment three electrode cell with a platinum disk/graphite paste as a working electrode, a platinum plate as an auxiliary electrode and $\mathrm{Ag} / \mathrm{AgCl}$ as a reference electrode. A $\lambda$-25 spectrophotometer of Perkin Elmer, Germany (obtained under DAAD Instrument Grant, Germany), was used for spectral analysis. Atomic force microscope (AFM) images were taken using AFM-STM (model PRO 47, NT-MDT, Russia).
Material was spin-coated over glass substrate through its THF solution and AFM images were taken in semicontact (tapping) mode. XRD and FT-IR characterizations were carried out with an $18 \mathrm{~kW}$ rotating anode Rigaku (Japan) powder X-ray diffractometer and a thermo model 5700 FT-IR, Germany, respectively. Thermogravimetric analysis (TGA) measurements were performed on a NETZSCH, STA 409 PC analyser with a heating rate of $10^{\circ} \mathrm{C} / \mathrm{min}$ under a flow of $\mathrm{N}_{2}$-gas. SEM was carried out at an operating voltage of $15-20 \mathrm{kV}$ using Shimadzu SS-550 Super Scan, Japan.

\subsection{Synthesis of PANA by emulsion polymerization technique}

Polymerization process was carried out in a $25 \mathrm{~mL}$ conical flask at room temperature $\left(25^{\circ} \mathrm{C}\right)$. Anthranilic acid monomer was dissolved in $12.5 \mathrm{~mL}$ chloroform (100 mM). To this solution, a solution of ammonium persulphate (APS) (400 mM) prepared in $2.5 \mathrm{~mL} 0.5 \mathrm{~N} \mathrm{H}_{2} \mathrm{SO}_{4}$ added dropwise with constant stirring. After $15 \mathrm{~min}$, a wine-coloured solution was seen, which further becomes a precipitate within $2 \mathrm{~h}$. The resulting solution was kept at $25 \pm 1^{\circ} \mathrm{C}$ overnight for complete polymerization. After $24 \mathrm{~h}$, a blackish brown precipitate was collected by centrifugation, followed by washing with $0.5 \mathrm{~N} \mathrm{H}_{2} \mathrm{SO}_{4}$ and finally with water. Polymer was dried under vacuum and the yield was calculated $(\sim 65 \%)$ to the weight of the monomer.

\subsection{Synthesis of PANA-Au nanocomposite by emulsion polymerization technique}

Polymerization process was carried out in a $25 \mathrm{~mL}$ conical flask at room temperature $\left(25^{\circ} \mathrm{C}\right)$. Anthranilic acid monomer was dissolved in $12.5 \mathrm{~mL}$ chloroform (100 mM). To this solution, a solution of gold chloride $(10 \mathrm{mM})$ prepared in $2.5 \mathrm{~mL} 0.5 \mathrm{~N} \mathrm{H}_{2} \mathrm{SO}_{4}$ was added dropwise with constant stirring. After $15 \mathrm{~min}$, a brownish precipitate was formed, which later consumed the entire solution within $2 \mathrm{~h}$. The resulting solution was kept at $25 \pm 1^{\circ} \mathrm{C}$ overnight for complete polymerization. After $24 \mathrm{~h}$, the purple brown precipitate was collected by filtration followed by washing with $0.5 \mathrm{~N} \mathrm{H}_{2} \mathrm{SO}_{4}$ and finally with water. The polymer formed was dried under vacuum and the yield was calculated ( $90 \%)$ to the weight of monomer and oxidizing agent weight.

\section{Results and discussion}

Chemical synthesis of PANA is scarcely reported in literature because of the high solubility of the polymer in common solvents. In our previous report, we mentioned about the synthesis method of PANA by using various oxidizing agents (Gupta and Prakash 2010). Degree of 

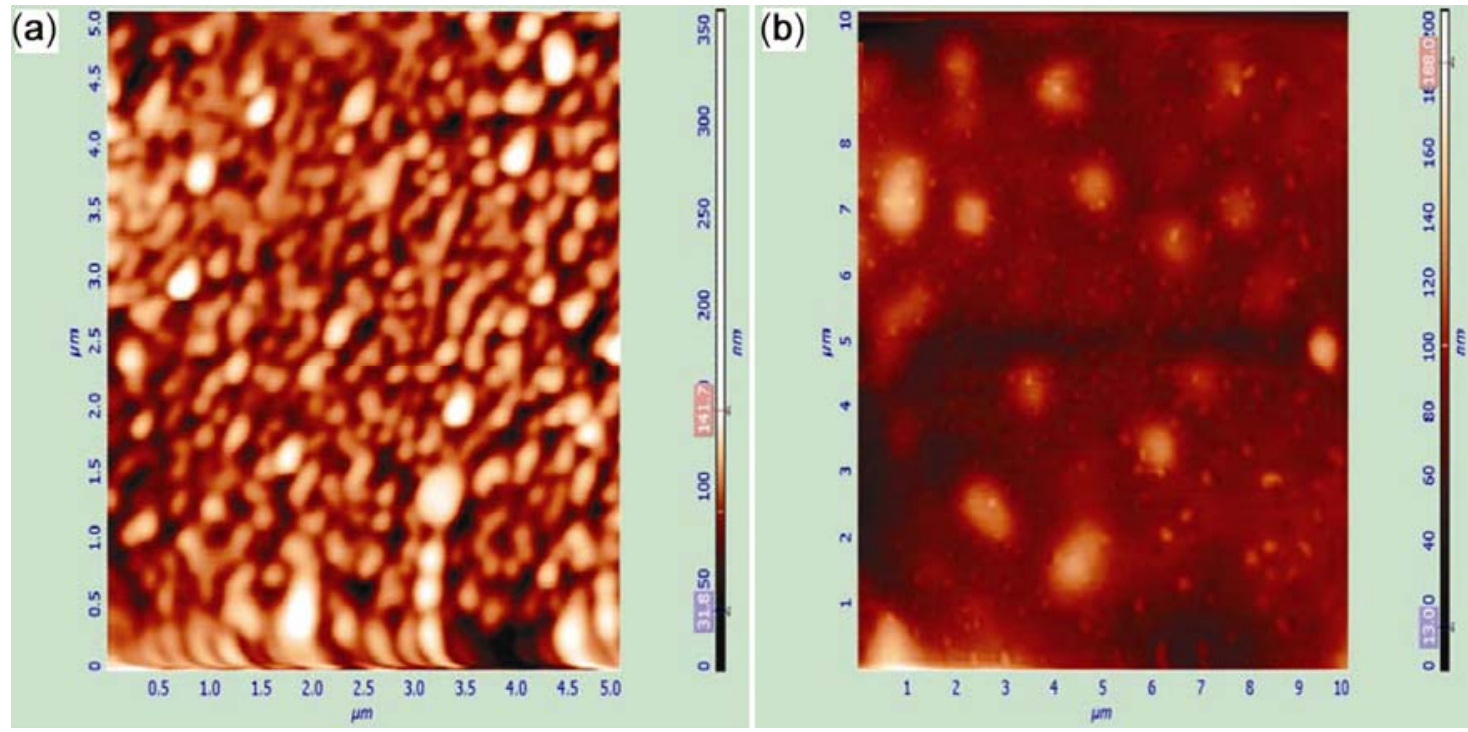

Figure 1. AFM images of (a) PANA and (b) PANA-Au nanocomposite.

chain length and various other properties were affected by different types of oxidizing agents. The above synthesis methodology is only limited to the synthesis of pristine polymer. By the use of gold chloride $\left(\mathrm{HAuCl}_{4}\right)$ as an oxidizing agent, the polymer formed with the modification is a nanocomposite. We report a simple method to synthesize $\mathrm{Au}$ nanoparticles with a reasonable size dispersity using water-soluble conducting polymer colloids of PANA. Mechanism of polymer formation from ions to gold particle $\left(\mathrm{Au}^{0}\right)$, which results in the growth, based on the reduction of Au polymer chain simultaneously. Thus, for the growing polymer chain, gold nanoparticles work as a reactive template; over which polymer grows. Au will either form tiny particles or it may collapse into one bigger particle and wrapped by polymeric chain. This depends on various parameters like oxidizing agent concentration, temperature and time of polymerization. Nanocomposite is highly dispersible in non-aqueous solvent that can be used for thin film formation. AFM image of a thin film polymer shows uniform polymeric globules and the nanocomposite depicting the presence of tiny nanoparticles in the polymeric matrix, as shown in figure 1 , with average surface roughness of 200 and $114 \mathrm{~nm}$, respectively. SEM images also support the presence of superficial Au nanoparticles. Morphology of polymeric globules is not very uniform in the case of nanocomposites opposite to that of pure polymer, probably because of the strong oxidizing nature of $\mathrm{HAuCl}_{4}$ than that of $\left(\mathrm{NH}_{4}\right)_{2} \mathrm{~S}_{2} \mathrm{O}_{8}$, as shown in figure 2. PANA-Au nanocomposite synthesized by emulsion polymerization route was studied for the reaction kinetics by UV-Vis spectroscopic method with various time intervals of polymerization. UV-Vis spectra of reaction medium were taken for various time interval, viz. $0,15,30 \mathrm{~min}, 1,2$ and $24 \mathrm{~h}$. In the case of emulsion polymerization with $\mathrm{HAuCl}_{4}$, a gradual fall in $\mathrm{HAuCl}_{4}$ absorbance was observed, as shown in figure 3. Complete disappearance of the $\mathrm{HAuCl}_{4}$ absorbance was observed after $30 \mathrm{~min}$. Furthermore, the growth of the polymer in case of nanocomposite was observed from the same technique and the resulted spectra are shown in figure 3 (inset). After complete disappearance of $\mathrm{HAuCl}_{4}$ absorbance, a clear feature of oligomeric or shorter-chain polymer was observed. First peak at around $320 \mathrm{~nm}$ corresponds to $\pi-\pi^{*}$ transition present in case of nanocomposite $(290 \mathrm{~nm}$ in case of polymer synthesized using ammonium persulphate), which is a clear indication of polymer growth with long conjugation length. A new peak was observed at around $440-455 \mathrm{~nm}$ after $30 \mathrm{~min}$, because of surface plasmon resonance of gold nanoparticles that merge with the polaronic transition of the polymer after $1 \mathrm{~h}$ of polymerization. Broadening in peaks observed in case of $2 \mathrm{~h}$ of polymerization depicts an immobilization of the polymer over gold nanoparticle. With further proceeding of polymerization, the peak shifted to higher wavelength region at $500 \mathrm{~nm}$. This could be because of polaronic transition of polymer (with longer chain length) and plasmonic transition of bigger Au nanoparticles. After completion of polymerization for $24 \mathrm{~h}$, the nanocomposite shows a bathochromic shift for all the transitions relative to that of pure polymer, as shown in figure 4, supporting long conjugation length and interaction between the $\mathrm{Au}$ and polymer.

The FT-IR spectra of the PANA and PANA-gold nanocomposites carried out in $\mathrm{KBr}$ matrix is shown in figure 5 . Features of the band are similar with shifting of the wave number to a lower value for the nanocomposite compared to that of pure polymer. The FT-IR spectra showed a characteristic peak at around $1686 \mathrm{~cm}^{-1}$ (absent in the PANi), which may be attributed to the strong 

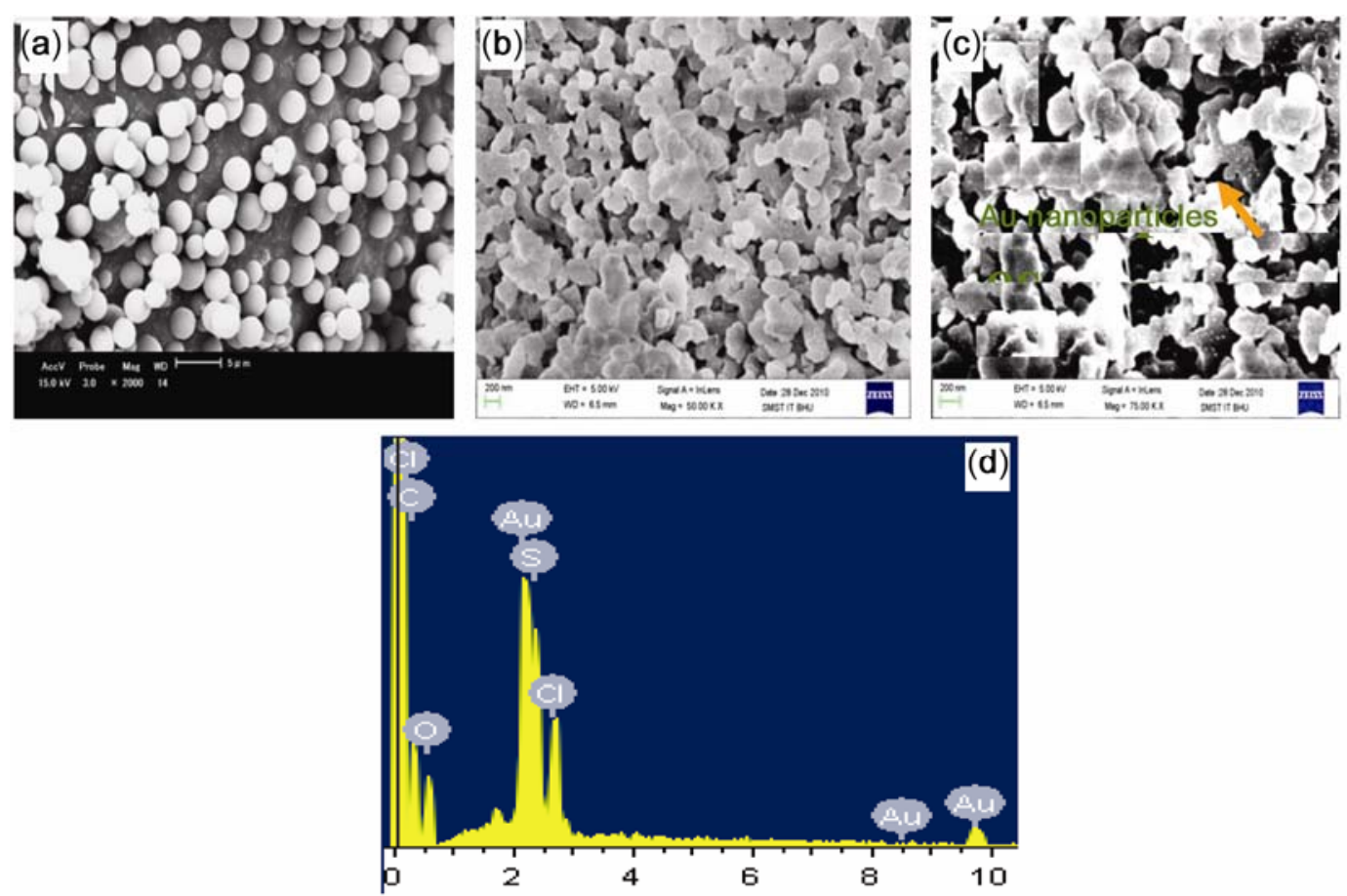

Figure 2. SEM images of (a) PANA, (b) PANA-Au nanocomposite, (c) higher magnified PANA-Au nanocomposite showing distributed Au nanoparticles and (d) EDAX data of PANA-Au nanocomposite.

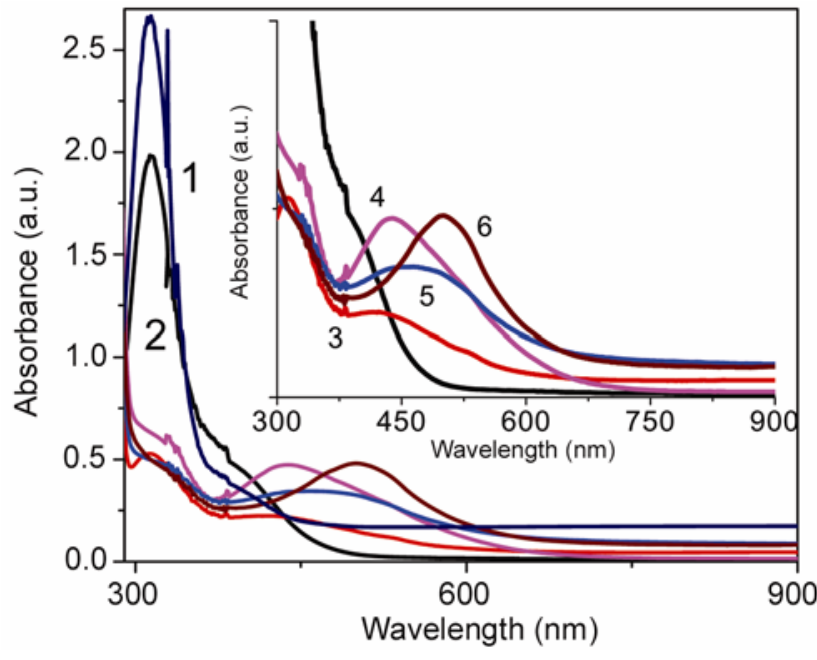

Figure 3. UV-Vis of (1) pure gold chloride and (2-6) anthranilic acid- $\mathrm{HAuCl}_{4}$ reaction mixture after (2) 15 min (3) $30 \mathrm{~min}(4) 1 \mathrm{~h} \mathrm{(5)} 2 \mathrm{~h}$ and (6) $24 \mathrm{~h}$.

stretching vibration of carboxyl group $(\mathrm{C}=\mathrm{O})$ in case of pure polymer synthesized using ammonium persulphate, and shifted to $1686 \mathrm{~cm}^{-1}$ in case of $\mathrm{Au}$ nanocomposite. The peak due to $\mathrm{C}=\mathrm{C}$ bond of benzenoid and quinoid rings are considered for the evaluation of the oxidation states and conjugation in the PANA formed by using ammonium persulphate and gold chloride. The $\mathrm{C}=\mathrm{C}$ stretching frequency of quinoid and benzenoid rings is observed at 1563 and $1505 \mathrm{~cm}^{-1}$ and 1563 and $1508 \mathrm{~cm}^{-1}$

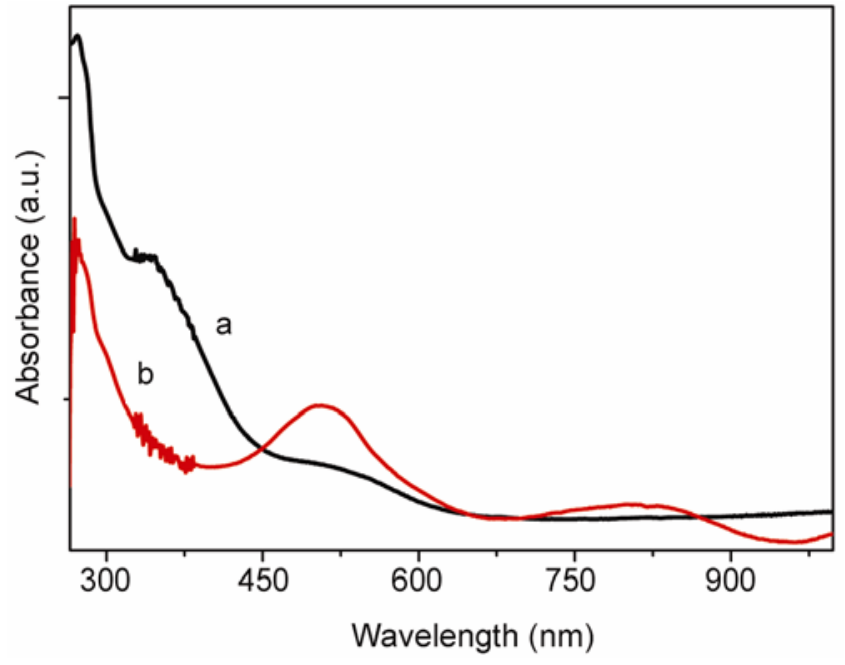

Figure 4. UV-Vis spectra of (a) PANA synthesized using (NH4) ${ }_{2} \mathrm{~S}_{2} \mathrm{O}_{8}$ and (b) PANA-Au synthesized using $\mathrm{HAuCl}_{4}$.

for the PANA and PANA-Au nanocomposite synthesized using emulsion polymerization route. Intensity ratio $\left(I_{\mathrm{Q}} / I_{\mathrm{B}}\right)$ of the band is also found to be higher in case of nanocomposite and, hence, supports higher oxidized state of polymer (Reddy et al 2008).

Redox property of PANA-Au nanocomposites was studied using differential pulse voltammetric technique. Equal amounts of nanocomposite were coated over platinum disc electrode by spin-casting method through THF 
solution. Two pairs of oxidation peaks are observed at 0.28 and $0.55 \mathrm{~V}$, which corresponds to leucomeraldine to emeraldine and emeraldine to pernigraniline transformations (Yue et al 1991; Li et al 2006) as shown in figure 6(a and $b$ ). The peak potential in case of nanocomposite is

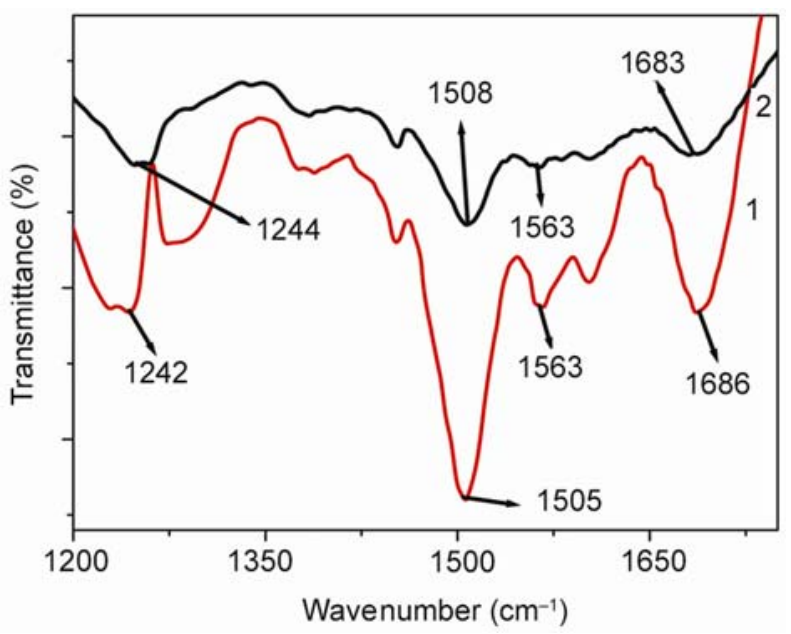

Figure 5. FT-IR of (1) PANA synthesized using $\left(\mathrm{NH}_{4}\right)_{2} \mathrm{~S}_{2} \mathrm{O}_{8}$ and (2) PANA-Au synthesized using $\mathrm{HAuCl}_{4}$.
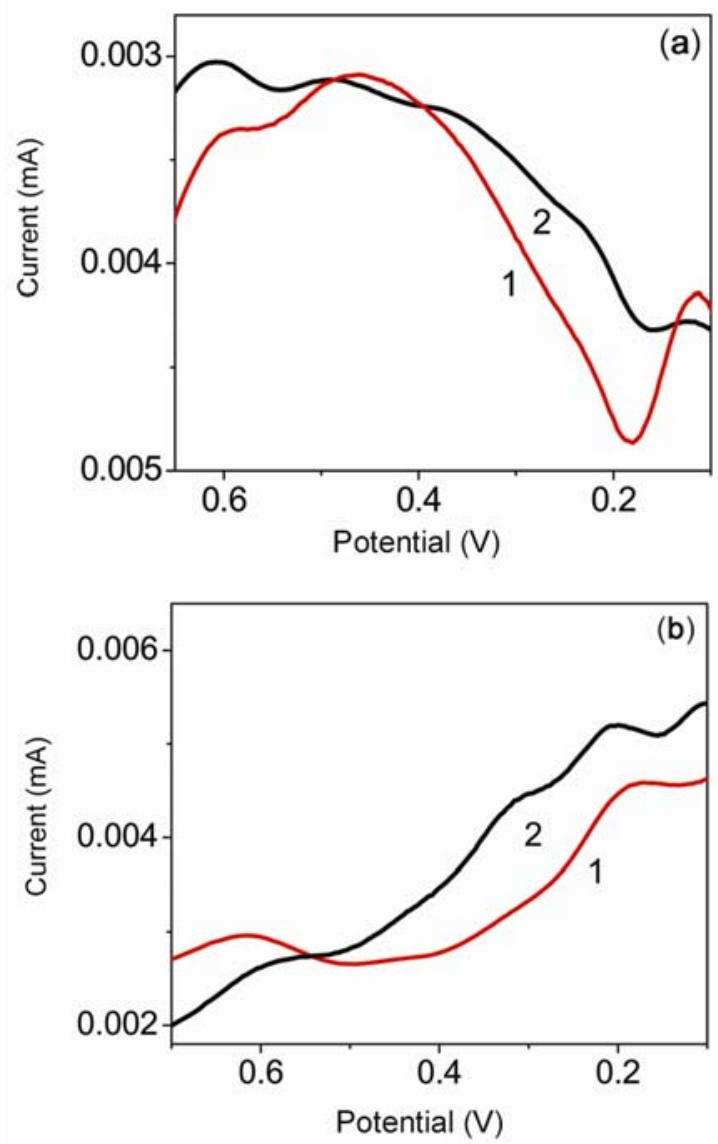

Figure 6. Differential pulse voltammetry (a) oxidation and (b) reduction of (1) PANA and (2) PANA-Au nanocomposite. lower than in the case of the pure polymer, probably because of facilitation of these transformations in the presence of Au particles. The relative density of both the transformations depicts a highly oxidized state of the polymer in the presence of $\mathrm{Au}$ nanoparticle, which is a clear indication of the charge transfer complex between nanoparticle and polymer. After comparing both the routes, it was found that PANA is more redox active and lesser oxidized than PANA-Au nanocomposite synthesized by emulsion route. This is clearly noticeable by the first redox transformation (not clearly visualized in case of nanocomposite) as shown in figure 6(a).

Formation of gold particles in PANA-Au composite material is highly phase selective as seen in powder XRD pattern (figure 7). A strong (1 111 ) Bragg reflection indicates that gold particles possess a highly oriented crystalline character confirming the face-centred crystal structure (Reddy et al 2008). Figure 7 (inset) indicates a less intense and amorphous behaviour of polymer in the
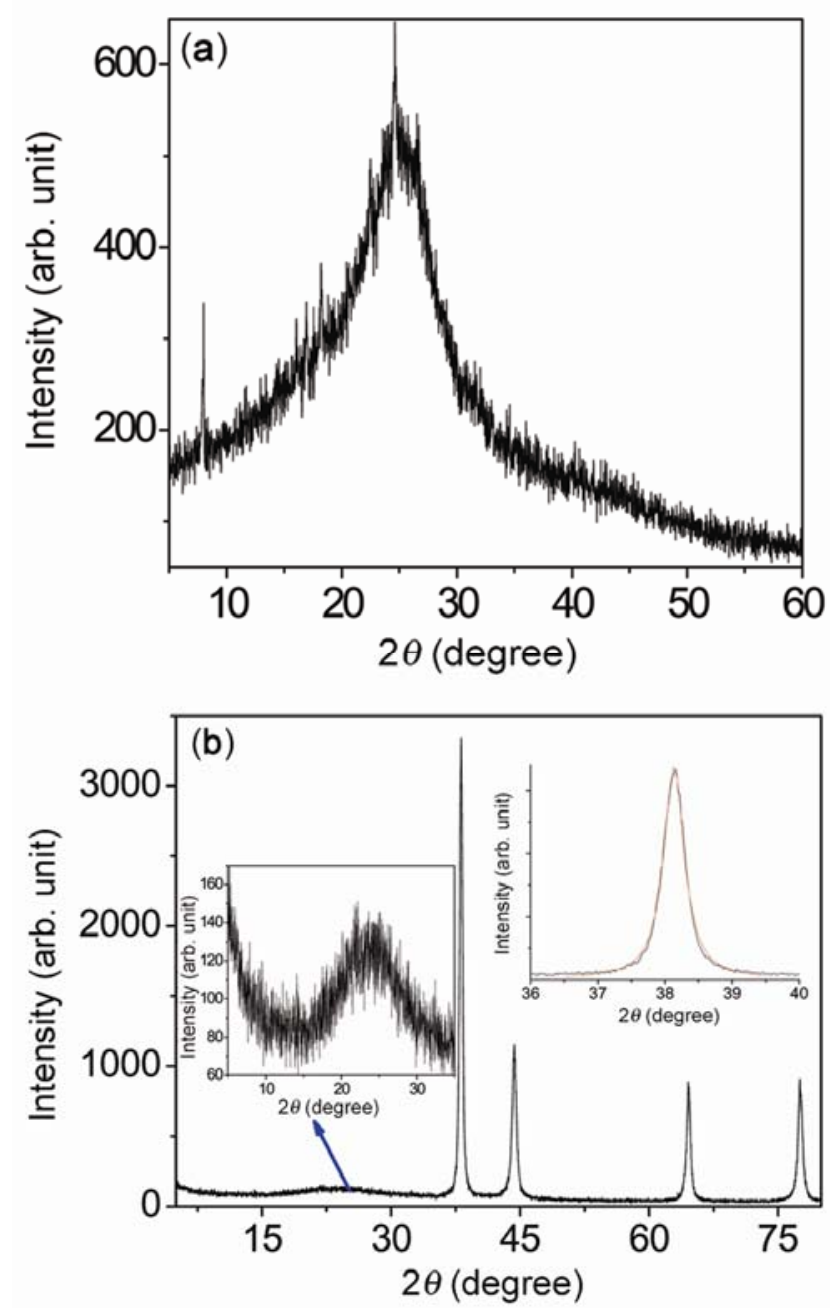

Figure 7. XRD pattern of (a) PANA and (b) PANA-Au nanocomposite Lorenzian best-fit curve for highest intensity peak (inset). 
lower $2 \theta$ range from 5 to $35^{\circ}$. Pure polymer shows a comparatively crystalline behaviour with an intense peak at around $7 \cdot 8^{\circ}$ that is because of polymeric self-assembly (Li et al 2008). The crystallite size of Au particle can be calculated by line broadening for the highest intense peak using Scherer's equation

$$
D=\frac{k \lambda}{\beta \cos \theta} .
$$

The average size of $\mathrm{Au}$ nanoparticles was calculated using (1) and estimated as $24.05 \mathrm{~nm}$ for nanocomposites synthesized by emulsion polymerization route. No sharp difference was observed in the diffraction pattern of the polymer.

Thermal behaviour of PANA shows a two-step weight loss process (Ogura et al 1991) and total weight loss reaches up to 35 and $58 \%$ of its original polymer quantity at $800{ }^{\circ} \mathrm{C}$ in case of PANA and PANA-Au nanocomposite, respectively as shown in figure 8 . The first weight loss at $180^{\circ} \mathrm{C}$ in case of pure PANA is attributed to the loss of first pair of carboxylic group. A shift of the same to a higher temperature in case of nanocomposite indicates a higher thermal stability of polymer in presence of $\mathrm{Au}$ nanoparticle. The second weight loss starting at around $300{ }^{\circ} \mathrm{C}$ in case of PANA-Au nanocomposite is assigned to the loss of second pair of carboxylic group followed by polymer chain degradation $\left(410^{\circ} \mathrm{C}\right)$. Two steps of carboxylic group degradation are clearly seen in case of nanocomposite probably because of gradual weight loss; however, in case of pure polymer, second carboxylic group degradation merges with the polymer degradation.

Figure 9(a) depicts the differential pulse voltammograms obtained at graphite paste electrode having $10 \mathrm{wt} \%$ of PANA-Au nanocomposite in $10 \mathrm{mM}$ Tris-HCl buffer

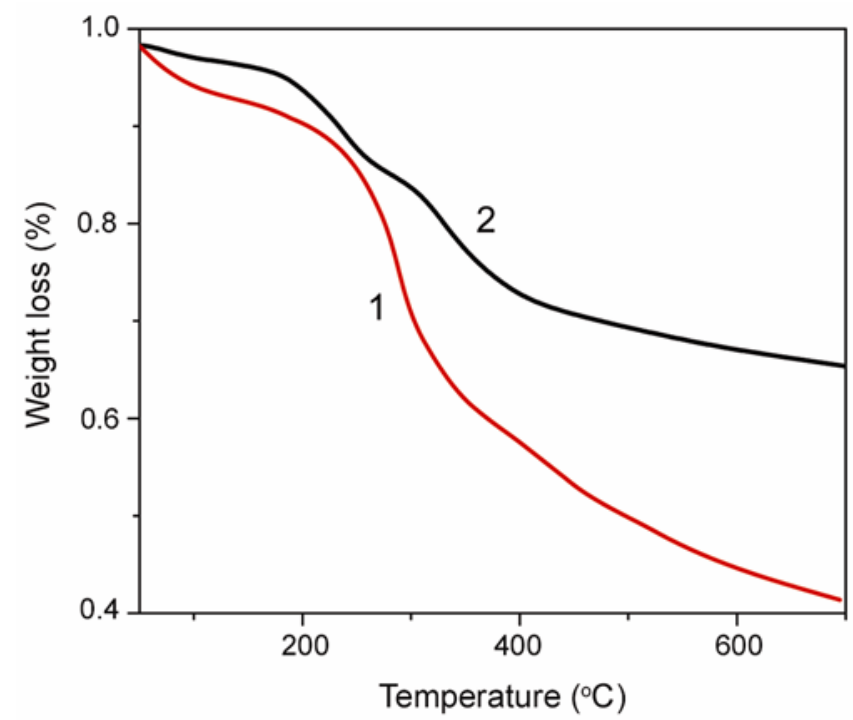

Figure 8. TGA of (1) PANA and (2) PANA-Au nanocomposite.
(pH 6.8) for different concentrations of DA from $10^{-6}$ to $10^{-3} \mathrm{M}$, where the concentration of AA was kept constant $(1 \mathrm{mM})$. It can be observed that almost no response occurs when $1 \mathrm{mM}$ AA was added in the Tris-buffer solution (curve a); this might be because of the acidic group $(-\mathrm{COOH})$ present on the polymer backbone. A sensitivity had been observed for AA on the polymer in the absence of carboxylic functional group. It was also observed that with an increase in DA concentration, oxidation current is also increased (represented by the headed arrow). It is clear that no oxidation peak for AA is present in case of graphite paste electrode modified with PANA-Au. While a broad oxidation peak for DA at $0.46 \mathrm{~V}$ was observed, suggesting the selectivity towards DA over modified electrode. The calibration curves for DA detection by DPV at the graphite paste electrode modified with PANA-Au were constructed using average currents recorded at three individual films for each concentration point. Figure 9(b) shows the calibration curves for DA
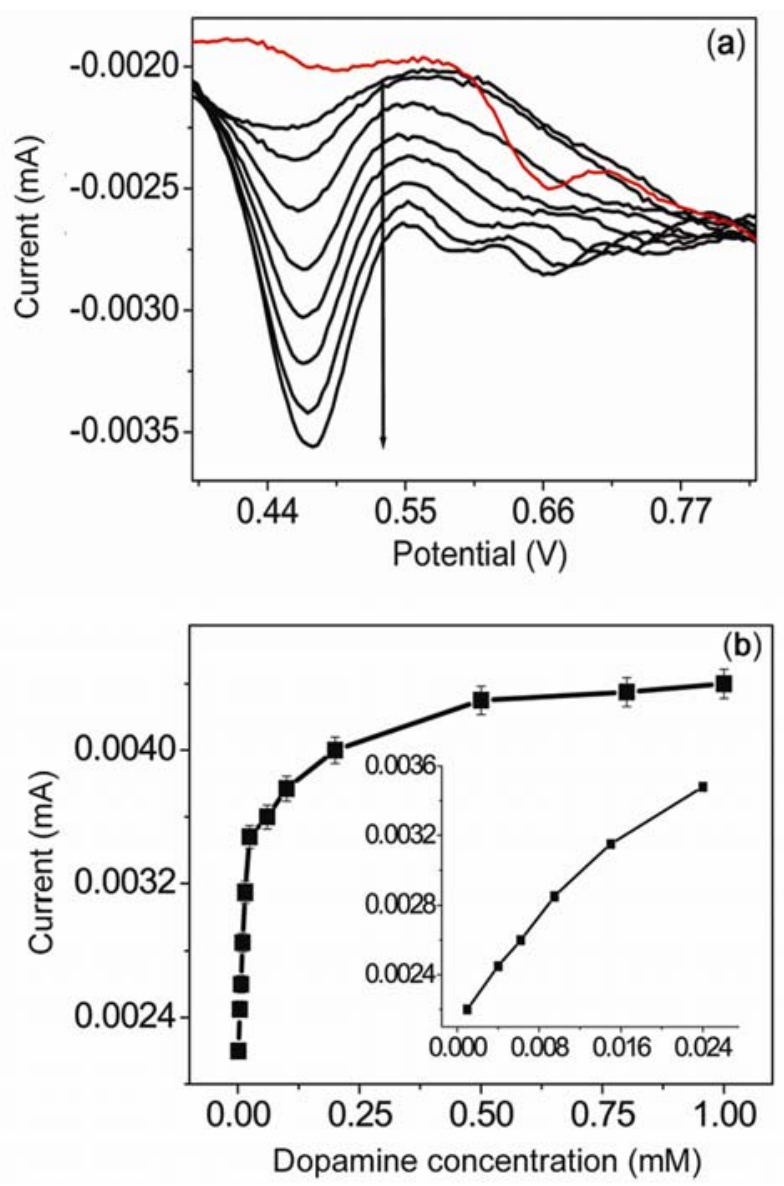

Figure 9. (a) Differential pulse voltammograms of graphite paste electrode modified with PANA-Au with $1 \mathrm{mM}$ AA followed by repeated additions of DA from $10^{-6}$ to $10^{-3} \mathrm{mM}$ in the presence of $1 \mathrm{mM} \mathrm{AA}$ in $10 \mathrm{mM}$ Tris- $\mathrm{HCl}$ buffer ( $\mathrm{pH} \mathrm{6.8)}$ ). (b) Calibration curve for the analysis of $10^{-6}-10^{-3} \mathrm{M}$ DA over graphite paste modified with PANA-Au (inset: linear part of the curve for sensitivity calculation). 
detection in presence of $1 \mathrm{mM}$ AA. The sensitivity towards DA sensing was found to be $12.5 \mu \mathrm{A} \pm 250 \mathrm{nA} / \mathrm{mM}$. Further, the lowest detection limit was found to be $1 \mu \mathrm{M}$. The enhanced sensitivity, improved linear response and better stability for DA detection without interference further proves its superiority to the earlier work (Mathiyarasu et al 2008) related to any conducting polymer$\mathrm{Au}$ modified electrode. The results support the usuability of these materials on small-size electrode for getting better sensitivity and selectivity for real-time estimation of DA.

\section{Conclusions}

Electroactive PANA with spherical morphology and globular PANA-Au nanocomposite was synthesized by two-phase polymerization through emulsion polymerization. Polymer formed by emulsion polymerization was similar to the polymer synthesized by conventional singlephase method, however, showed spherical morphology. Nanocomposite synthesized using $\mathrm{HAuCl}_{4}$ having globular morphology with uniformly distributed gold nanoparticles, which reduced the band gap of the polymer and increased thermal property drastically. Nanocomposite of PANA with Au nanoparticles was used for making highly stable, AA interference-free dopamine sensor with sensitivity and lower detection limit of $12.5 \mu \mathrm{A} \pm$ $250 \mathrm{nA} / \mathrm{mM}$ and $10^{-6} \mathrm{M}$, respectively. Studies on miniaturized electrode for in vivo studies is under progress.

\section{Acknowledgement}

Authors are thankful to Prof D Panday, SMST, IIT, BHU, for providing XRD facilities and DST, New Delhi, for Inspire Faculty Fellowship.

\section{References}

Banik R M, Mayank, Prakash R and Upadhyay S N 2008 Sens. Actuat. B: Chem. 131295

Boal K, Ilhan F, DeRouchey J E, Thurn-Albercht T, Russell T P and Rotello V M 2000 Nature 404746
Dewald J L, Wondmagegn W T, Ellis A V and Curran S A 2005 Synth. Met. 15539

Downard A J, Roddick A D and Bond A M 1995 Anal. Chim. Acta 317303

Gupta B and Prakash R 2009 J. Mater. Sci. Eng. C29 1746

Gupta B and Prakash R 2010 Polym. Adv. Technol. 211

Hable C T and Wrighton M S 1991 Langmuir 91305

Jin J, Manoharan M P, Wang Q and Haque M A 2009 Appl. Phys. Lett. 95033113

Li J, Jia Q, Zhu J and Zheng M 2008 Polym. Int. 57337

Li W, Jia Q X and Wang H L 2006 Polymer 4723

Maheshwari V and Saraf R F 2006 Science 3121501

Majumdar G, Castro E G, Canestraro C D, Zanchet D, Ugarte D, Roman L S and Zarbin A J G 2006 J. Phys. Chem. B110 17063

Majumdar G, Goswami M, Sarma M T K, Paul A and Chattopadhyay A 2005 Langmuir 211663

Mallick K, Witcomb M J and Scurrell M S 2006 J. Mater. Sci. 416189

Mallick K, Witcomb M, Scurrell M and Strydom A 2009 J. Phys. D: Appl. Phys. 42095409

Mathiyarasu J, Senthilkumar S, Phani K L N and Yegnaraman V 2008 Mater. Lett. 62571

Melvin A, Vijay R, Chaudhari V R, Gupta B, Prakash R, Haram S, Baskar G and Khushalani D 2010 J. Coll. Inter. Sci. 346265

Mirkin C A and Letsinger R L 1996 Nature 382607

Mohan S and Prakash R 2010 J. Mater. Sci. Eng. C30 781

Nielsen C B and Bjørnholm T 2005 Macromolecules 3810379

Ogura K, Shiigi H, Nakayama M and Ogawa A 1999 J. Polym. Sci. Part A: Polym. Chem. 374458

Pandey P C, Chauhan D S and Singh V 2009 Electrochim. Acta 542266

Power A C, Betts A J and Cassidy J F 2010 Analyst 131645

Reddy K R, Lee K P, Lee Y and Gopalan A I 2008 Mat. Lett. 621815

Selvaraj V and Alagar M 2008 Nanotechnology 19045504

Singh A K, Joshi L, Prakash R and Kaneto K 2010 Japanese J. Appl. Phys. 49 01AD06

Singh A K, Prakash R, Dwivedi A D D and Chakrabarti P 2008 Synth. Metals 158939

Tseng R J, Huang J, Ouyang J, Richard B, Kaner R B and Yang Y 2005 Nano Lett. 51077

Tu X, Xie Q, Jiang S and Yao S 2007 Biosens. Bioelectron. 22 2819

Yue J, Wang Z H, Cromack K R, Epstein A J and MacDiarmid A G 1991 J. Am. Chem. Soc. 1132665 